\title{
NUMERICAL WAVE DISSIPATION OVER IDEALIZED MARSH PLATFORM
}

\author{
Elizabeth R. Holzenthal ${ }^{1}$ and David F. Hill ${ }^{2}$
}

\begin{abstract}
Combined tidal and wave events are simulated over an idealized marsh environment to quantifiably characterize the cross-shore attenuation of wave height as a function of bottom roughness and submergence depth. Dissipation calculated by the numerical model is compared with existing analytically derived parametric wave height decay models. We propose an alternative two-parameter dissipation model that better captures the limited decay of wave attenuation over a kilometer of propagation. The results suggest that cross-shore decay rates are sensitive to incident wave conditions as well as bottom roughness.
\end{abstract}

Keywords: wave attenuation by vegetation; bottom roughness dissipation; spectral wave model; parametric decay model

\section{BACKGROUND}

Hydrodynamic interactions between coastal vegetation, overland flow, and wave transformation are complex phenomena. Although there has been increased study of these interactions at smaller scales (see Anderson et al. (2011) and Wu et al. (2011) for comprehensive reviews), appropriately resolving vegetation is a remaining challenge for regional scale coastal models commonly used for long-term planning and risk assessment. Due to computational constraints, hydraulic models tend to incorporate vegetation with empirically derived representative bottom roughness coefficients, such as Manning's n parameter (Bunya et al. 2010; Passeri et al. 2011). Manning's n parameterizes the bed shear stress term in Generalized Wave Continuity Equation (GWCE) for the Advanced Circulation (ADCIRC) model, and it is used to calculate the wave friction factor $f_{w}$ in the spectral wave model SWAN when the two are coupled (Luettich et al. 1992; Dietrich et al. 2011). This study seeks to examine the effect of spatial variability on the attenuation of spectral wave height $H$ over an idealized tidal marsh profile as computed by the coupled ADCIRC+SWAN numerical model. To characterize the dissipative capacity of modeled roughness configurations, we turn to existing analytical models of wave height transform over vegetation.

For the case of normally incident non-breaking waves of unit width propagating over a rough, nonsloping bottom boundary for a duration $T$ and traveling a distance $L$, we can assume that the rate of energy $E$ transfer from time $t_{0}$ and location $x_{0}$ to $t_{0}+T$ and $x_{0}+L$ is balanced by the spatial gradient in energy flux $\mathcal{F}$ and the time-averaged rate of energy dissipation $\epsilon_{d}$ due to bottom friction

$$
\frac{d E}{d t}=\frac{d \mathcal{F}}{d x}+\epsilon_{\mathrm{d}}
$$

The time-averaged rate of dissipation can be thought of as the phase-averaged product of bed shear stress $\tau_{\infty}$ and the velocity at which this stress is applied, approximated as the free stream velocity at the turbulent boundary layer $\mathrm{u}_{\infty}(\mathrm{t})$, where the subscript $\infty$ is used to mean very near the turbulent boundary layer (Nielsen 1992). Combining the expression for energy flux $\mathcal{F}=E C g$ and the expression for $\epsilon_{d}$ by Jonsson (1966), we can express the time-averaged energy balance as

$$
\frac{d}{d x}\left(\frac{1}{8} \rho g H^{2} \frac{\sigma}{k} n\right)=-\frac{2}{3 \pi} \rho f_{w}(A \sigma)^{3}
$$

where parameters $\rho$ represent water density, $g$ acceleration due to gravity, $\sigma$ angular frequency, $k$ wavenumber, $n$ the ratio of group to phase speed, and $A$ the wave orbital radius at the turbulent boundary layer, equivalent to $H / 2 \sinh (k h)$. Thus, we can simplify Eq. 2 to

$$
\frac{d H^{2}}{d x}=\frac{H}{2} \frac{d H}{d x}=-C H^{3}, \quad C=\frac{2}{3 \pi} \frac{f_{w} k \sigma^{2}}{g n \sinh ^{3} k h}
$$

\footnotetext{
${ }^{1}$ School and Civil and Construction Engineering, Oregon State University, 1491 SW Campus Way, Corvallis, OR 97331, United States; holzente@oregonstate.edu

2 Professor, School and Civil and Construction Engineering, Oregon State University, 1491 SW Campus Way, Corvallis, OR 97331, United States
} 
Solving this differential equation and imposing the incident boundary condition $H(x=0)=H_{0}$, we find that the cross-shore attenuation of wave height $H(x)$ has the form

$$
H(x)=\frac{H_{0}}{1+K_{1} x}, \quad K_{1}=\frac{4}{3 \pi} \frac{H_{0} f_{w} k \sigma^{2}}{g n \sinh ^{3} k h}
$$

This hyperbolic decay curve is consistent with the analytical model of wave attenuation by vegetation derived by Dalrymple et al. (1984). Applying conservation of energy for the case of waves propagating through a field of stems idealized as rigid cylinders, the dissipation rate $\epsilon_{d}$ is found to be the product of the wave velocity $u$ and the resulting drag force imposed on the cylinders $F_{D}$. The resulting attenuation model is identical that of Eq. 4, but the decay rate $K_{1}$ is a function of the geometric properties and arrangement of the cylindrical stems rather than a bulk coefficient.

Taking a different approach, Kobayashi et al. (1993) again treat vegetation as rigid cylinders but instead apply conservation of momentum to derive an exponential expression of wave height attenuation,

$$
H(x)=H_{0} \exp \left(-K_{2} x\right)
$$

Many field and lab studies of wave transformation through vegetation have relied on this model to quantify dissipation rates, including but not limited to Möller et al. (1999), Anderson and Smith (2014), and Bradley and Houser (2009). Numerous others report non-linear attenuation of waves across marshes, with wave height decay rates greatest within the first few meters of vegetation patch (Möller and Spencer 2002; Knutson et al. 1982; Anderson et al. 2011).

Classifying vegetative resistance with macroscale cross-shore decay rates has the advantage of essentially collapsing a two-parameter problem (i.e. how much attenuation occurs over what spatial extent?) into a single parameter, which can more readily be related to input variable conditions. We take this approach in evaluating the sensitivity of numerical wave attenuation by varying configurations of bottom roughness as well as submergence depth. We begin by comparing the results of numerical simulations with existing parametric models of wave attenuation. The best-fitting analytical model is then parameterized with modeled observations, and a relationship between decay coefficients and bottom roughness configurations is proposed.

\section{METHODS}

\section{Experimental Design}

Tide and wave events are simulated for the case of an idealized tidal wetland using the coupled ADCIRC+SWAN coastal circulation and spectral wave model. Morphological features include of a lowsloping foreshore, a planar tidal platform elevated above mean sea level $(z=0.5 \mathrm{~m})$, and a sloping backshore. The full domain, shown in Figure 1, is $3 \mathrm{~km}$ in length and $250 \mathrm{~m}$ in width; mesh resolution is uniform throughout the domain with average node spacing of $15 \mathrm{~m}$.
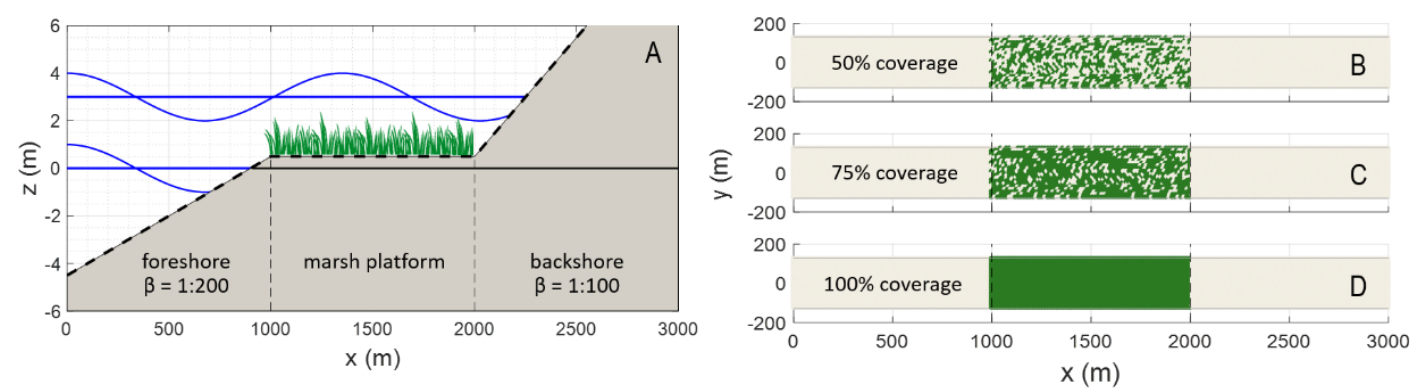

Figure 1. Idealized tidal platform used in numerical experiment. (A) Plan view showing morphological features and tidal range during low water $(M S L=0 \mathrm{~m})$ and high water $(M S L=3 \mathrm{~m}$ ) simulations; constant spectral wave forcing is imposed at the offshore boundary. Grass shown only to indicate region of idealized vegetation. Aerial view of enhanced bottom friction distributed randomly at $50 \%$ (B), $75 \%$ (C), and $100 \%$ (D) spatial density on the planar marsh platform.

Three environmental conditions are varied in the model to study their impact on wave attenuation by vegetation: density of spatial coverage, degree of roughness, and depth of submergence. To examine 
the effect of vegetation spatial coverage, enhanced bottom friction is distributed at random to $50 \%, 75 \%$, and $100 \%$ of nodes within the elevated vegetated platform. Degree of roughness is achieved by increasing bottom roughness in regions designated as vegetated from a Manning's $n$ value of 0.02 to an extreme value of 0.35 . A value of 0.02 , representative of a smooth, sandy bottom, is used as a background value in regions not designated vegetated. Table 1 shows the Manning's n values used in the experiment and their corresponding representative vegetation type (Bunya et al. 2010).

\begin{tabular}{|l|l|}
\hline \multicolumn{2}{|l|}{$\begin{array}{l}\text { Table 1. Manning's } \mathbf{n} \text { bottom roughness coefficients, } \\
\text { representing idealized vegetation. }\end{array}$} \\
\hline Manning's n & Land cover description \\
\hline 0.020 & Smooth, sandy bottom \\
0.035 & Saline marsh \\
0.070 & Wetland scrub \\
0.105 & Saltmarsh, upper limit \\
0.140 & Wetland forest \\
0.175 & Upland forest \\
0.210 & Forest, upper limit \\
0.350 & Unrealistic \\
\hline
\end{tabular}

We consider the sensitivity of wave attenuation to submergence depth by simulating two combined wave-tidal events. In both experiments, we impose tidal forcing (amplitude of $1 \mathrm{~m}$ ) for the duration of our simulation; the forced wave conditions at the domain boundary are set to a $3 \mathrm{~m}$ significant wave height $H$ and peak period $T_{p}$ of $10 \mathrm{~s}$. In the first case, the marsh platform sits $0.5 \mathrm{~m}$ above mean sea level (MSL), which is a reasonable approximation for this environment (Loder et al. 2009). For the second case, we elevate the MSL to be $2.5 \mathrm{~m}$ above the marsh platform but maintain all other forcing conditions. Simulations are run for a two-day period to allow for gradual ramping of boundary forcing so as to avoid numerical instabilities at the wetting and drying landward boundary. Covering a range of eight Manning's n values, three coverage densities, and two water depths, a total of forty-eight simulations were performed with the dynamic coupled model.

Steady-state SWAN simulations were also performed to isolate the effect of bottom roughness from other variables, namely dynamic water depths and a non-uniform bed profile. The static simulations were performed for all eight Manning's $\mathrm{n}$ parameters exclusively at full coverage of the marsh platform. Consistent with the dynamic simulations, wave forcing boundary conditions of $3 \mathrm{~m} \mathrm{H}$ and $10 \mathrm{~s} T_{p}$ were used. Four static water depths were considered, ranging from $0.5 \mathrm{~m}$, equivalent to high tide during the low-water dynamic forcing case, increasing in 1-m intervals to $3.5 \mathrm{~m}$, equal to high tide during the highwater dynamic simulation.

\section{Analysis Approach}

For each simulation, unique in its combination of spatial coverage, degree of roughness, and hydrodynamic forcing, we isolate $H(x)$ profiles from the centerline of the marsh platform and normalize it by the incident wave height $H_{0}$. Wave profiles are extracted in this manner from the dynamic coupled simulations when the average water depth across the tidal platform is equal to $0.5 \mathrm{~m}, 1.5 \mathrm{~m}, 2.5 \mathrm{~m}$ and $3.5 \mathrm{~m}$ in order to examine sensitivity to submergence depth. The resulting cross-shore profiles are then fit to the hyperbolic and exponential decay models. Additionally, we assess the applicability of a third parametric model,

$$
H(x)=H_{0}[(1-a) \exp (-b x)+a]
$$

This modified exponential curve essentially imposes a limit to exponential attenuation; for very small $a$ values, the expression is identical to the decay model of Kobayashi et al. (1993), but for nonzero $a$ and very large distances $x$, wave height approaches an asymptotic fraction of $H_{0}$. Goodness of model fit to observed data, here referring to cross-shore profiles of simulated $H / H_{0}$, is quantified with root mean square error (RMSE). We also consider how closely the wave height at the end of the platform is predicted by the three parametric attenuation models.

\section{RESULTS}

Wave profiles across enhanced bottom friction, simulated by the numerical model ADCIRC+SWAN, were compared to analytical models of wave attenuation. For each simulation, we calculate the error between the simulated attenuation profile $H(x) / H_{0}$ and that calculated from the 
hyperbolic, exponential, and modified exponential model. In our inter-model comparison, we use only full-coverage bottom friction configurations. Additionally, it was observed that model fit error was less sensitive to variable Manning's n than submergence depth of the marsh platform. Thus, an average RMSE across all input bottom friction values is used represent a single metric of model fit to observed wave transformation given a particular water depth. Figure 2 shows the results of our error analysis from two perspectives.

First, we quantify the mismatch between the simulated and parametrically modeled attenuation across the entire cross-shore profile, shown on the left-hand side. Fig. 2A shows the mean RMSE associated with fitting wave profiles simulated with the coupled ADCIRC+SWAN model to the hyperbolic (red), exponential (blue), and modified exponential (green) parametric models as a function of relative wave height $(H / h)_{0}$ incident to the vegetated region. Dashed lines of the same color scheme are used to show one standard deviation from the mean RMSE. Secondly, we calculate the bias in the simple parametric models in estimating the wave height at the end of the vegetated platform. Bias of the three simple models, quantified as percent increase from the landward wave height numerically modeled by the coupled model, is shown in Fig. 2B. As seen in Figs. 2A and B, the modified exponential equation yields minimized RMSE and narrowest deviation from simulated landward wave heights across submergence depths.
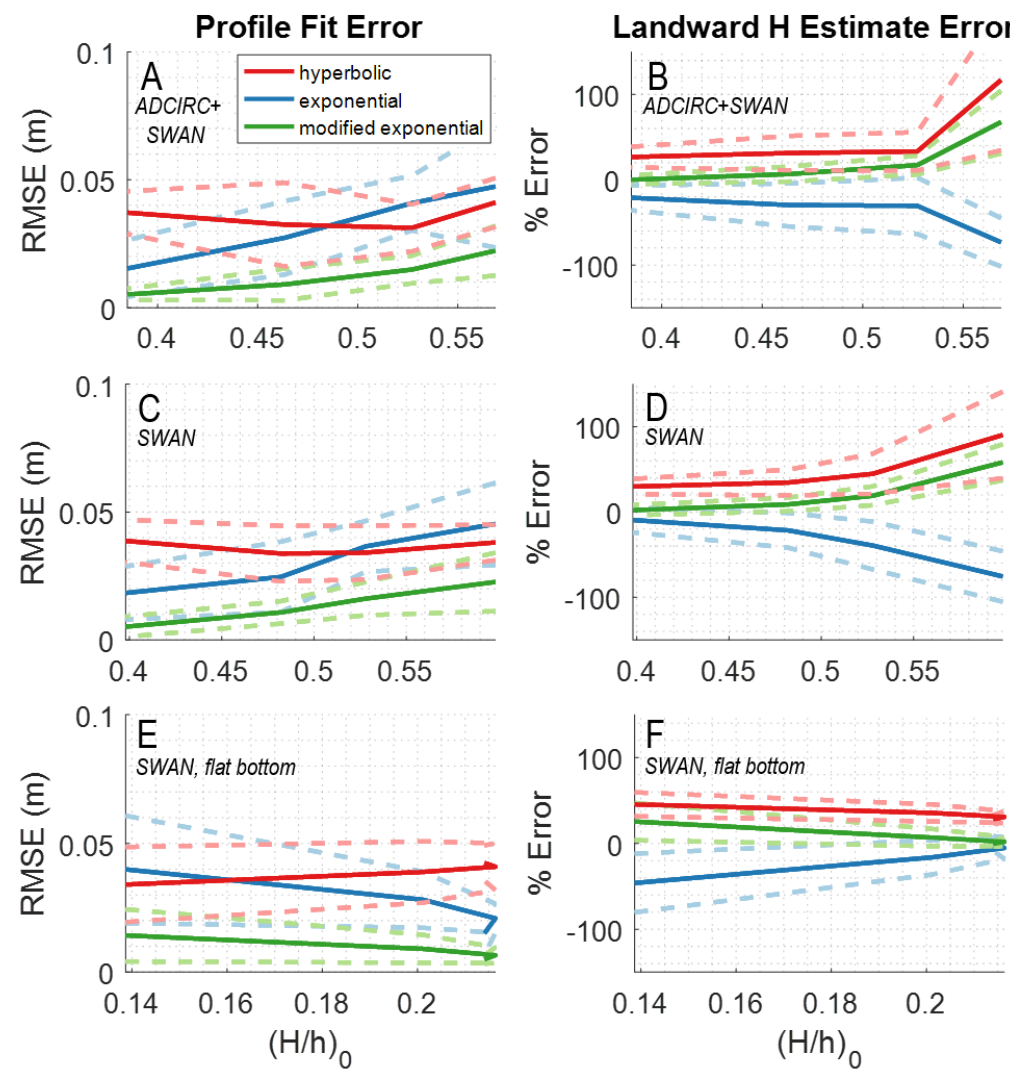

Figure 2. Comparison of numerically and parametrically modeled wave height attenuation profiles (A, C, E) and wave height at the end of the vegetated region (B, D, F). Solid lines indicate average error metric for all bottom roughness values (full coverage of marsh platform); dashed lines indicate uncertainty at one standard deviation from the mean. Error analysis was performed using results of the dynamic, coupled model $(A, B)$ as well as for the standalone wave model with idealized marsh bathymetry (C, D) and with a uniform bottom of identical length $(E, F)$.

Though this study is primarily concerned with wave dissipation under combined wave and tide hydrodynamics, we also examine the possibility that dynamic water depths are responsible for the poorness of fit for the analytical hyperbolic and exponential attenuation models. Standalone SWAN was therefore used to transform wave heights over the idealized platform at static water depths equal to those isolated from the dynamic model ( $h=0.5 \mathrm{~m}$ and increasing $1-\mathrm{m}$ intervals). Wave transform over a 
bathymetrically constant domain with identical water depths and boundary wave forcing was also calculated using SWAN to eliminate shoaling effects near the seaward boundary of the marsh platform. The results of model fit analysis using uncoupled SWAN are shown in Figs. 2C and D for the idealized marsh profile and Figs. $2 \mathrm{E}$ and $\mathrm{F}$ for the uniform depth simulations. As with the coupled model, the twoparameter modified exponential model yields less error than the hyperbolic and simple exponential models; the improved behavior of this model is consistent whether estimating wave attenuation profiles or wave heights at the end of the marsh platform.

Returning to the results of the dynamically coupled model, we now focus on our second objective: characterizing the relationship between incident wave conditions, bottom roughness configuration, and attenuation. Combinations of Manning's $n$ and percent spatial coverage are consolidated into a single parameter, a spatially averaged Manning's n coefficient, or effective Manning's n. Figures 3A and C show that with increasing bottom roughness, the spatial decay rate $b$ increases and the attenuation limit $a$ decreases. Both parameters vary greatly with effective Manning's $\mathrm{n}$ at lower values and appear to level out as roughness values increase. This asymptotic behavior is generalized with hyperbolic tangent trend lines, which proved to best fit the spread of $a$ and $b$ parameters. The average r-squared $\left(r^{2}\right)$ of the individual trend lines for data grouped by water depths was 0.77 for the $a$ parameter and 0.55 for the $b$ parameter. Due to the high degree of scatter from water depth variability, the $r^{2}$ values of the full dataset trends are much lower.

The relationship between water depth and decay parameters are shown in Figs. 3B and D. For lowest submergence depths $(h=0.5 \mathrm{~m})$, incident relative wave height varied widely with no apparent pattern. Thus, the resulting decay parameters from low-water simulations were excluded from the calculation of the incident-condition trend line across the dataset. As a result, a first order polynomial was chosen as a representative trend; decay parameters are tightly clustered around few $(H / h)_{0}$ value, leaving effectively three data points from which to draw inference.
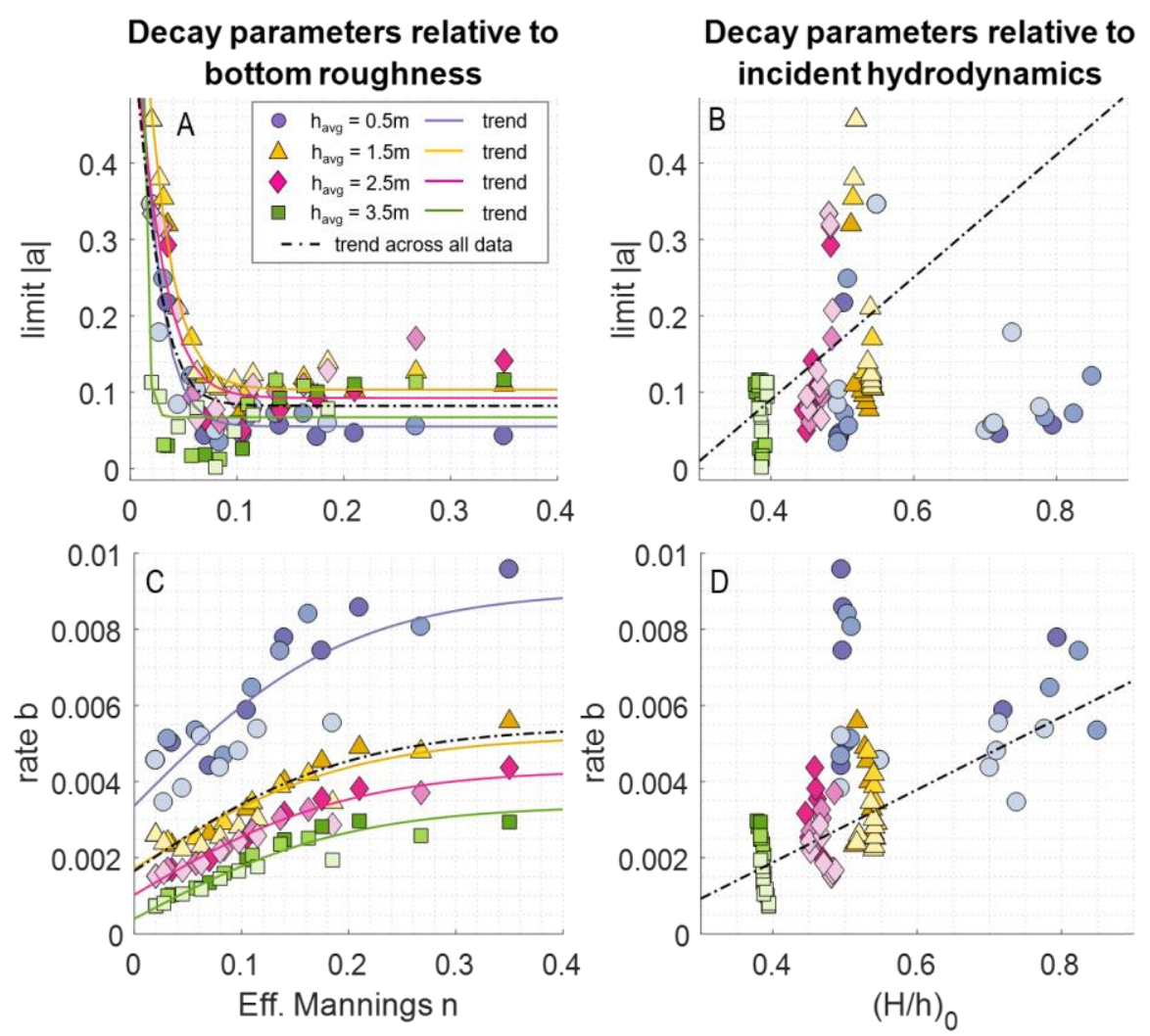

Figure 3. Relationship between wave decay parameters $a$ and $b$ and effective bottom roughness coefficient (A, C) and incident relative wave height (B, D). Marker indicates wave attenuation parameterization corresponding to particular instances of average water depth on the platform. Solid lines represent best-fit trend for conditions of corresponding marker color. Dot-dash black line represents trend across all data. 


\section{CONCLUSIONS}

Provided they can be properly validated, parametric models of wave transformation can offer an immediate first-order approximation of wave dissipation by vegetation. This study makes an initial effort in calibrating attenuation equations from numerical experiments of waves over varying bottom roughness configurations and at various water depths. Though several other decay equations generally capture the nonlinear behavior of wave dissipation by idealized vegetation, e.g. those by Dalrymple et al. (1984) and Kobayashi et al. (1993), we find that the numerically simulated wave profiles most closely follow a modified exponential decay model (Eq. 6). The profile-wise comparison in Fig. 2 shows that the goodness of fit of the two-parameter decay model is consistent across the range of hydrodynamic conditions and computational schemes tested. The very small difference in averaged RMSE fit metrics between Figs. 2A-B and C-D is due to fact that spatial variability in water depth during dynamic simulations was not very large. Also of note is that incident conditions were significantly smaller during the depth-uniform static SWAN simulations (Figs. 2E-F). Unlike waves propagating through the idealized marsh domain, wave dissipation by bottom roughness, even without enhanced roughness, is not counteracted by shoaling before reaching the vegetated platform. Consequently, the SWAN simulations with a flat bottom essentially extend the range of incident wave conditions over which the parametric model analysis is considered; even at very low incident relative wave conditions, the twoparameter model yields less RMSE.

Model bias is more readily perceived at a single location. The two-parameter decay model was originally proposed because the simple exponential model tended to greatly underestimate dissipation in the beginning of the marsh platform and overestimate total wave attenuation. Thus, it is important to note that the one-parameter decay model is considered a conservative predictor of wave height after a kilometer of propagation, but the same is not necessarily true if estimating wave attenuation over a significantly smaller region of vegetation (Figs. 2B, D, F). In addition to the bathymetric variability at the seaward boundary of the marsh platform, the topographic gradient at the landward boundary may also influence parametric model fit results. At higher water levels, waves propagate across the domain with reduced turbulent bottom boundary interactions and are thus larger at the intersection of the planar vegetated region and sloping backshore. Wave shoaling along the sloping landward boundary is likely the cause for the large increase in error at higher incident wave conditions (Figs. 2B and D).

There are many interesting relationships evident in Figure 3. Sensitivity to incident wave conditions is evident in subfigures A-D, though results suggest that the offset parameter $a$ may be less sensitive to incident conditions than decay rates $b$. From two different perspectives, there seems to be a limit on total wave attenuation modeled by the spectral wave model SWAN. From a parametric model perspective, we see that the limited-exponential model is best exemplified by low-roughness conditions; waves do not decay exponentially ad infinitum, particularly when bottom boundary dissipation is minimal. Secondly, the limited nature of wave dissipation evident by these results is shown also at high bottom roughness conditions. There does appear to an upper threshold of bottom roughness above which wave attenuation no longer increases. The role of spatial variability on total dissipative capacity is largely dependent on the resulting effective bottom roughness. In this study, spatial variability is portrayed as "patchiness" of vegetation, ultimately lowering the spatially averaged Manning's n. In other cases, spatial variability may increase roughness of a region predominantly covered by a less rough feature, which may enhance wave height attenuation. Further, the relationship between bottom roughness and cross-shore decay parameters are clearly nonlinear (Figs. 3A and C).

This work is very noticeably neglecting a few key elements. A significant oversimplification made by this study of wave dissipation by vegetation is the uncoupling of vegetation and bathymetry, which has been shown to be intimately interdependent (Marani et al. 2013). Applying a range of friction values representing characteristic vegetation without incorporating correspondingly representative geomorphic settings is not realistic. However, isolation of one feature does allow more tractable attribution of attenuation to vegetation itself (though itself simplified as 'roughness') rather than to the combined vegetation-bathymetric effect. It is also important to note that wave-tidal interactions are not fully captured in SWAN's bottom roughness dissipation formulation; SWAN does not incorporate current velocities in its calculation of wave friction factor though $f_{w}$ is a function of $\mathrm{u}_{\infty}$. These inconsistencies are among other avenues of investigation we aim to further pursue.

\section{ACKNOWLEDGEMENTS}

This work is supported by the Graduate Research Fellowship Program awarded by the National Science Foundation and the Karen Irons Medicis Scholarship awarded by the ARCS Foundation Oregon Chapter. 


\section{REFERENCES}

Anderson, M.E. and J.M. Smith. 2014. Wave attenuation by flexible, idealized salt marsh vegetation. Coastal Engineering, 83:82-92.

Bradley, K. and C. Houser. 2009. Relative velocity of seagrass blades: Implications for wave attenuation in low-energy environments. Journal of Geophysical Research: Earth Surface, 114(F1).

Bunya, S., J.C. Dietrich, J. Westerink, B. Ebersole, J. Smith, J. Atkinson, R. Jensen, D. Resio, R. Luettich, C. Dawson, et al. 2010. A high-resolution coupled riverine flow, tide, wind, wind wave, and storm surge model for southern Louisiana and Mississippi. Part I: Model development and validation. Monthly Weather Review, 138(2):345-377.

Dalrymple, R.A., J.T. Kirby, and P.A. Hwang. 1984. Wave diffraction due to areas of energy dissipation. Journal of Waterway, Port, Coastal, and Ocean Engineering, 110(1):67-79.

Dietrich, J.C., M. Zijlema, J.J. Westerink, L.H. Holthuijsen, C. Dawson, R.A. Luettich, R.E. Jensen, J.M. Smith, G.S. Stelling, and G.W. Stone. 2011. Modeling hurricane waves and storm surge using integrally-coupled, scalable computations. Coastal Engineering, 58(1):45-65.

Jonsson. I.G. 1966. Wave boundary layers and friction factors, paper presented at the 10th International Conference on Coastal Engineering, ASCE, Tokyo.

Knutson, P.L., R.A. Brochu, W.N. Seelig, and M. Inskeep. 1982. Wave damping in Spartina alterniflora marshes. Wetlands, 2(1):87-104.

Kobayashi, N., A.W. Raichle, and T. Asano. 1993. Wave attenuation by vegetation. Journal of Waterway, Port, Coastal, and Ocean Engineering, 119(1):30-48.

Loder, N.M., J.L. Irish, M.A. Cialone, and T.V. Wamsley. 2009. Sensitivity of hurricane surge to morphological parameters of coastal wetlands. Estuarine, Coastal and Shelf Science, 84(4):625636.

Luettich, R.A., J.J. Westerink, and N.W. Scheffner. 1992. ADCIRC: An Advanced Three-Dimensional Circulation Model for Shelves, Coasts, and Estuaries. Report 1. Theory and Methodology of ADCIRC-2DDI and ADCIRC-3DL. Technical report, Coastal Engineering Research Vicksburg, MS.

Marani, M., C. Da Lio, and A. D’Alpaos. 2013. Vegetation engineers marsh morphology through multiple competing stable states. Proceedings of the National Academy of Sciences, 110(9):32593263.

Möller, I. and T. Spencer. 2002. Wave dissipation over macro-tidal saltmarshes: Effects of marsh edge typology and vegetation change. Journal of Coastal Research, 36(sp1):506-521.

Möller, I., T. Spencer, J. R. French, D. Leggett, and M. Dixon. 1999. Wave transformation over salt marshes: a field and numerical modelling study from North Norfolk, England. Estuarine, Coastal and Shelf Science, 49(3):411-426.

Nielsen, P. 1992. Coastal Bottom Boundary Layers and Sediment Transport. World Scientific, Singapore.

Passeri, D., S.C. Hagen, D. Smar, N. Alimohammadi, A. Risner, and R. White. 2011. Sensitivity of an ADCIRC tide and storm surge model to Manning's n. Estuarine and Coastal Modeling, 457-475.

W. Wu, Y. Ozeren, D. Wren, Q. Chen, G. Zhang, M. Holland, Y. Ding, S. Kuiry, M. Zhang, R. Jadhav, et al. 2011. Phase I Report for SERRI Project No. 80037: Investigation of surge and wave reduction by vegetation. Technical report. 